\title{
An Overview of Shoulder Instability and its Management
}

\author{
Nicholas Greville Farrar, Joby Jacob George Malal*, Jochen Fischer and Mohammed Waseem
}

\author{
Macclesfield District General Hospital, Victoria Road, Macclesfield, Cheshire, SK10 3BL, UK
}

\begin{abstract}
The assessment and management of patients with instability of the shoulder joint can be challenging, due to the varying ways patients present, the array of different classification systems, the confusing terminology used and the differing potential management strategies. This review article aims to provide a clear explanation of the common concepts in shoulder instability and how they relate to the assessment and management of patients.

There are sections covering the mechanisms of shoulder stability, the clinical assessment of patients and imaging techniques. Beyond that there is a discussion on the common classifications systems used and the typical management options.

Some patients fall into reasonably well defined categories of classification and in these cases, the management plan is relatively easy to define. Unfortunately, other patients can elude simple classification and in these instances their management requires very careful consideration. Further research may help to facilitate a better understanding of management of the patients in this latter group.
\end{abstract}

Keywords: Shoulder, instability, dislocation, shoulder arthroscopy, subluxation.

\section{INTRODUCTION}

Shoulder instability is a broad topic, encompassing frank dislocation of the gleno-humeral joint requiring formal reduction, subtle symptoms and demonstrable clinical signs. Several classification systems have been suggested, yet it has not proved easy to guide management based on these classifications. Many protocols for the non-operative rehabilitation of patients with shoulder instability have been devised, along with many surgical techniques. Controversy still exists as to which management strategy is best in each situation.

This article will describe the normal anatomical stabilisers of the shoulder joint, the clinical assessment of patients, use of imaging, the aetiology and classification of shoulder instability and some of the common management options.

\section{MECHANISMS OF SHOULDER STABILITY}

The shoulder joint affords a great freedom of motion in all planes, which in combination with the elbow allows us to place our hands into the position of maximum function for the task required. This freedom of movement comes at a price, with the shoulder joint being the most commonly dislocated joint in the body. An elaborate system of both static and dynamic stabilisers maintains stability and keeps the humeral head centred in the glenoid throughout the full range of physiological motion.

\footnotetext{
*Address correspondence to this author at the Department of Orthopaedics, Macclesfield District General Hospital, Victoria Road, Macclesfield, Cheshire, SK10 3BL, UK; Tel: +44 1625 661315; Fax: +44 1625 425873; E-mail: jjgeorgemalal@gmail.com
}

The static stabilisers include the glenoid labrum, the capsule-ligamentous thickenings and the inherent negative intra-articular pressure maintained by the joint capsule. These static restraints are particularly important at the extremes of motion, with varying contributions depending on the position of the humeral head. The glenoid labrum itself is not a large structure, but does deepen the glenoid 'socket' for which the bony component alone is very shallow and inherently unstable. This structure will be discussed in more detail in the management section.

Anterior subluxation of the humeral head is resisted by capsular thickenings between the glenoid and the humerus. Of particular importance are the superior gleno-humeral ligament (SGHL), the middle gleno-humeral ligament (MGHL) and the inferior gleno-humeral ligament (IGHL). The ligaments become taught at the extremes of motion with varying degrees of importance depending on the amount of shoulder abduction and external rotation. When the shoulder is adducted and in neutral rotation, hanging by the side of the chest, the SGHL will be taught and prevent inferior subluxation $[1,2]$. The negative intra-articular pressure may also contribute to this [3]. As the shoulder joint abducts, the prime static restraint transfers from the SGHL to the MGHL and by 45 degrees of abduction, the MGHL will be most important [4]. At higher degrees of abduction the IGHL assumes greater importance as a static stabiliser, and in particular the anterior bundle of the IGHL. There are both anterior and posterior bundles, forming the anterior and posterior boundaries of the axillary pouch. This is often referred to as a hammock that supports the humeral head in abduction, since it was described by O'Brien [5].

Concavity-compression is a description of the way in which the dynamic stabilisers of the shoulder joint function. 
The principle is that muscular contraction compresses the humeral head into the glenoid, centring it and increasing the force required to translate the humeral head out of this centred position. With the arm held adducted by the chest wall in the neutral position, the forces produced by deltoid contraction act to pull the humeral head vertically in the line of its muscle fibres. This can be seen clinically in patients with major deficiency of the rotator cuff, who demonstrate this 'shrug' of the shoulder when trying to initiate shoulder abduction, as the humeral head simply abuts under the coraco-acromial arch. In a normal shoulder the rotator cuff musculature will centre the humeral head according to the concavity-compression principle, facilitating the action of the powerful deltoid muscle, once the supraspinatus has initiated the first few degrees of elevation. As abduction increases the force vector produced by the deltoid increases concavity-compression, up to a maximum at 90 degrees.

\section{CLINICAL ASSESSMENT}

Commonly there will have been a clear history of a traumatic dislocation of the shoulder, followed by a closed manipulation in the emergency department, with confirmatory radiographs. In this case it is clear where to focus the clinical assessment. However when there has not been a defined single episode of trauma, it is useful to maintain a high index of suspicion that instability may be the diagnosis.

There are many useful pieces of information to gather in the clinical history. If dealing with a young patient in their $2^{\text {nd }}$ or $3^{\text {rd }}$ decade then instability is a relatively common cause of shoulder pain. If the patient is a keen sportsman in this age group then superior labral anterior to posterior (SLAP) tears can be either a differential diagnosis or an associated pathology, particularly in those taking part in overhead throwing sports. Other differentials in this age group could be pain from a previous injury to the acromioclavicular joint, or osteolysis of the lateral end of the clavicle in a young weight lifter. In the $4^{\text {th }}$ and $5^{\text {th }}$ decades, alternative diagnoses become more common such as subacromial impingement syndrome, frozen shoulder and inflammatory arthritis. Beyond that in the $6^{\text {th }}$ decade and beyond, tears of the rotator cuff and degenerate joint disease figure more highly in the list of probabilities. Age, therefore can be of use when hypothesis testing in the history, but is of course just a guide and there is overlap in the ages at which the various pathologies present.

The occupation and hobbies of the patient are very useful to note, as it gives information of potential mechanisms of injuries and a guide to the functional demands on the shoulder. Additionally, a keen sportsman is likely to have different expectations of the potential management strategy than someone with a more sedentary lifestyle.

If there has been an injury to the shoulder then this needs exploring. It needs to be determined if there a documented dislocation of the shoulder and if so, was it anterior or posterior? What position was the arm held in at the time of dislocation and how violent was the injury? It is useful to know if a closed reduction was performed in the emergency department or if reduction was performed in the operating theatre with a documented examination under anaesthesia post reduction. It must be ascertained if this was the first episode of dislocation, or if there have been other episodes.

Whether there was a landmark traumatic event in the history or something much more subtle, patients may complain of on-going pain with certain activities or with the arm in certain positions. For example, anterior instability may produce pain, or just a subjective feeling of instability, when the arm is in an abducted and externally rotated posture. The patient may actively avoid this posture or precipitating activity. 'Dead arm syndrome' has been described in which patients complain of their arm 'going dead' or 'lame' after a forceful overhead activity such as a tennis serve for example [6]. This results in the patient ceasing activity. Some patients will be aware of the sensation of subluxation, but some will not and so it is in this group that a high index of clinical suspicion has to be maintained.

It is useful to know if the patient has had any physical therapy since the episode, what this has involved and whether there has been any response to this. If any surgery has been undertaken then full details must be obtained before any consideration could be given to any revision procedure. Symptoms of generalised ligamentous laxity should be enquired upon, along with the other components of a full general clinical history.

After taking a history, it is useful to start with some of the pieces of the examination that can be performed with the patient still sitting in their chair. The Beighton score is a useful measure of generalised ligamentous laxity and whilst sitting it is possible to check if the thumbs passively flex to the volar aspect of the forearm and if the little fingers passively dorsiflex beyond 90 degrees each [7]. A point is awarded for each of these features if demonstrated. The elbows can then be checked for hyperextension. If they extend to beyond 10 degrees past neutral then a point is awarded for each elbow. Whilst remaining in the chair the knees can also be checked for hyperextension beyond 10 degrees. The final and potential ninth point in the Beighton score requires the patient to then stand and attempt to bring their palms flat onto the floor with the knees remaining hyperextended. A score of 4 or greater is generally regarded as significant.

Once standing it is useful to observe how easy (or not) it is for the patient to remove their outer garments to expose the upper limbs and trunk. Inspection may reveal asymmetry, prominence of the acromio-clavicular joint, clavicular malunion, or wasting of the supraspinatus and/or infraspinatus in their relevant fossae. A prominent biceps muscle belly ('pop-eye') sign indicates rupture of the long head of biceps. Palpation for tenderness can then be carried out in a systematic way, although this is not often demonstrated in cases of instability. The authors' preference is to start at the sterno-clavicular joint and then progress along the subcutaneous border of the clavicle onto the acromio-clavicular joint, before palpating around the acromion, where tenderness may be demonstrated in cases of impingement syndrome. The gleno-humeral joint lines may then be palpated anteriorly and posteriorly.

Standing in front of the patient it is easy to demonstrate the shoulder movements in sequence, whilst observing the patient's active range of motion. If there is any deficit, then 
it should be ascertained if the full passive range is achievable. The rotator cuff muscles should then be assessed individually against resistance and compared to the opposite side. Jobe described the empty can test for supraspinatus function. The elbows are extended and the shoulders abducted to 90 degrees and brought forward by 30 degrees into the plane of the scapula with the shoulders internally rotated so that the thumbs point to the floor [8]. The patient is asked to maintain this position whilst the examiner exerts downward pressure on the forearms, starting with gentle pressure and increasing the force as desired. With this test weakness of the supraspinatus can be demonstrated, or alternatively power may be preserved but the manoeuvre is painful, which may indicate that a partial tear is present. Infraspinatus function should be assessed with resisted external rotation, with the shoulder adducted, whilst palpating the muscle belly in the infra-spinous fossa. If there is clear weakness then the test should be repeated with the shoulder abducted to 90 degrees and externally rotated fully. Failure of the patient to maintain this position, constitutes the Hornblower's sign and indicates that in addition to a tear of the infraspinatus, there is likely to also be a tear of the teres minor. Subscapularis should be assessed with the belly press test, in which the patient places both hands onto their abdomen and then moves their elbows forward as the subscapularis internally rotates the shoulder. If this is easily demonstrated then light resistance can be applied against the elbows to compare the two sides. Aggressive resistance to this movement is not useful as patients will then recruit their pectoralis major and anterior deltoid muscles. Gerber's lift off test essentially excludes a large tear of the subscapularis and is tested by asking the patient to place the dorsum of their hand onto their lumbar region and then push their hand away dorsally [9].

A great number of special tests have been described relating to shoulder instability; however, the clinical history should direct attention to those which are likely to be most relevant in each case. Our practice is to start by trying to demonstrate a sulcus sign, with the patient standing up and the arm adducted by the patient's side [10]. A hand is placed onto the upper trapezius and gentle downward pressure is applied to stabilise the shoulder girdle, whilst the other examining hand grasps the arm and applies a distracting force. If there is global laxity, then a sulcus appears inferior to the lateral edge of the acromion. Whilst the patient is still standing, an anterior apprehension test can be performed. It is useful for the patient to stand in front of a mirror, so that the examiner can see their face whilst standing behind the patient. To examine the left shoulder, the right examining hand is placed onto the top of the shoulder to stabilise the scapula, whilst the left hand holds the patient's elbow. The shoulder is brought up to 90 degrees of abduction with the elbow also flexed to 90 degrees. The examiner's right thumb applies a gentle pressure on the posterior humeral head whilst the shoulder is slowly externally rotated. A sensation of apprehension/ instability or an anterior pain has been described as a positive result and the shoulder should be brought back to neutral at this point [11].

With the patient lying on the examination couch in the supine position, the apprehension test can be repeated by again bringing the shoulder to 90 degrees abduction with a flexed elbow and then gradually externally rotating the shoulder. If apprehension is demonstrated, then the other examining palm can be placed over the humerus to provide a posteriorly directed force. This is a positive anterior apprehension/ relocation test if the patient's discomfort is relieved with this posterior force [12]. Still in the supine position, the shoulder can be flexed to 90 degrees and internally rotated. Posteriorly directed axial force in this position can elicit the sensation of instability in a positive posterior apprehension test [13]. Sometimes with this test, the humeral head can be felt to sublux posteriorly and then relocated if the shoulder is abducted and externally rotated. Finally, it can be useful to perform anterior and posterior draw test in the supine position to further assess the directions of any laxity. The examiner's hand grasps the scapula posteriorly and it's coracoid process anteriorly whilst the other hand cradles the proximal humerus, with the thumb anteriorly and the other fingers posteriorly. Anterior and posteriorly directed forces can then be applied to assess for any humeral head translation. The degree of translation can be graded, with grade 0 indicating no detectable translation, grade 1 indicating slightly increased joint laxity and grade 2 is recorded when the humeral head is felt to shift over the glenoid rim, indicating a significant degree of joint laxity [14].

\section{DIAGNOSTIC IMAGING}

In the acute traumatic presentation, plain radiographs are usually sufficient to guide the initial management. A standard series in our institution includes an anterior to posterior (AP) view of the gleno humeral joint, an axial view and a scapula ' $Y$ ' view. These views should be enough to determine if there is a dislocation and, if there is, to describe in which direction. Fig. (1a, b) show a typical anterior shoulder dislocation. All but the most subtle of proximal humerus fractures can also be detected with these views. It is vital to obtain radiographs prior to any attempt to reduce a shoulder dislocation. If a displaced proximal humerus fracture is identified on a post reduction radiograph, in the absence of pre reduction views, it is difficult to dispute an allegation that the attempted reduction manoeuvre displaced what was previously an undisplaced fracture.

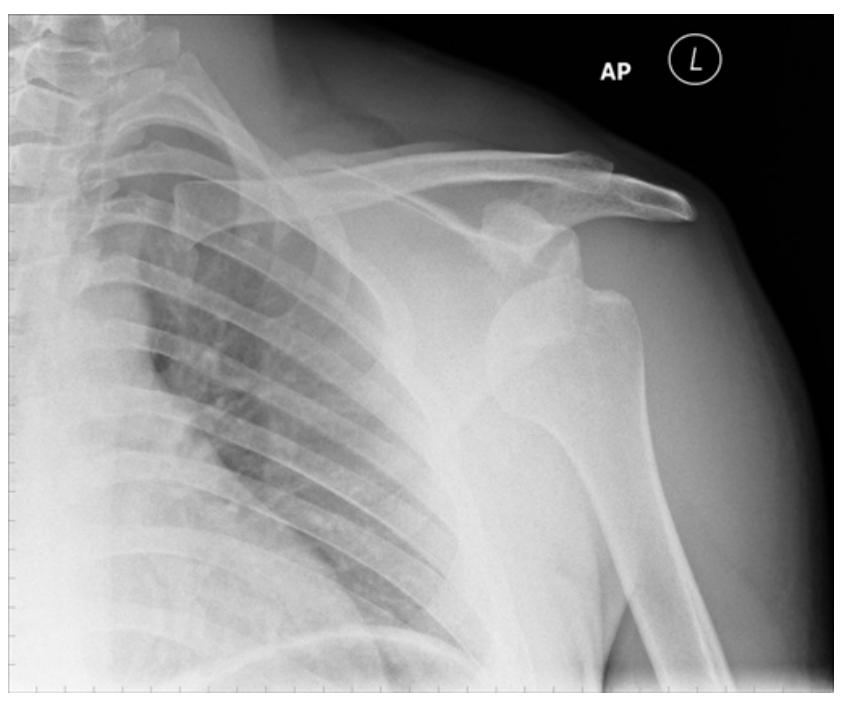

Fig. (1a). This AP radiograph of the shoulder demonstrates a typical anterior dislocation. 


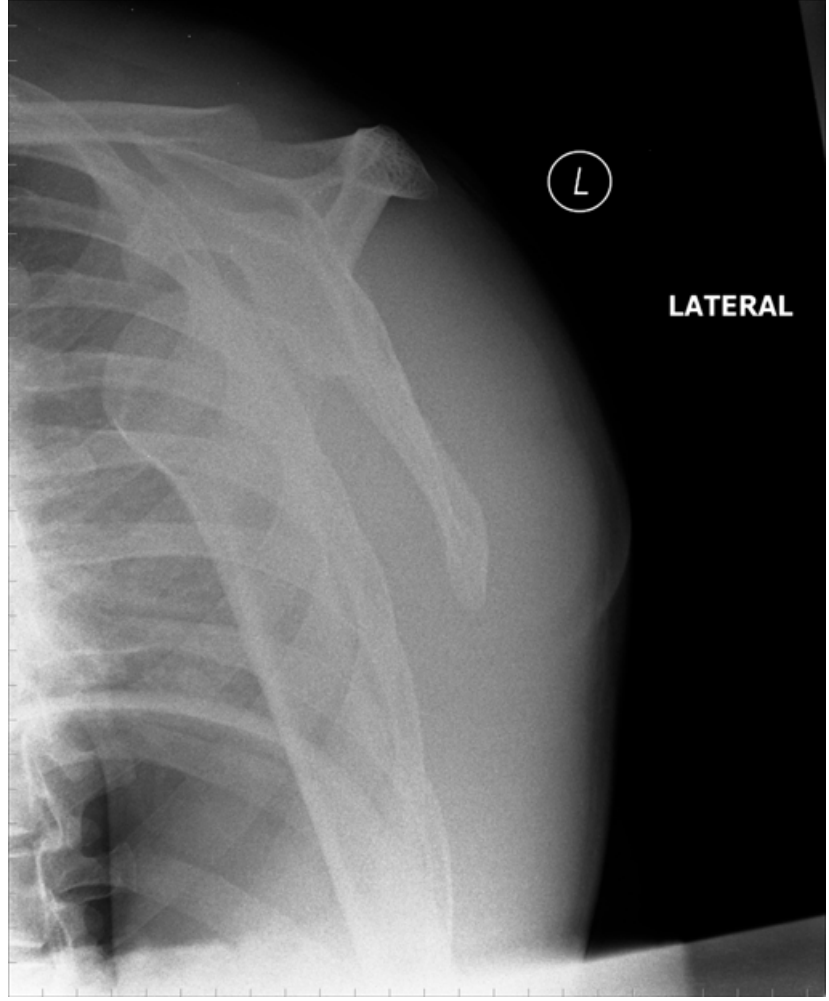

Fig. (1b). This scapula ' $Y$ ' view confirms the findings from figure xa.

Plain radiographs can identify a glenoid fracture, in which case, further imaging in the form of a CT scan is usually indicated. A 'bony' Bankart lesion can sometimes be seen. This is a bony sign to indicate the presence of a capsule-ligamentous detachment from the anterior glenoid, described by Bankart in his classic paper in 1938 [15]. A small bony lesion can be seen in the region of the posterior glenoid rim and this is often referred to as a 'reverse' Bankart lesion, on the basis that it resembles the classic Bankart lesion, but is associated with a posterior dislocation.

On a post reduction radiograph, as well as examining for a Bankart lesion, the outline of the humeral head should be observed to look for a bony defect resulting from a compression fracture as the humeral head levers against the glenoid rim during dislocation. Such a compression fracture of the postero-lateral humeral head is described as a HillSachs lesion and is associated with an anterior dislocation [16]. A similar compression fracture located anteriorly on the humeral head and associated with a posterior dislocation, is often revered to as a 'reverse' Hill-Sachs lesion.

Computed tomography (CT) is particularly useful when determining if there has been significant glenoid bone loss, either acutely with a large Bankart lesion, or in chronic cases of instability. Axial cuts can help to quantify bone loss in cases of chronic instability, providing useful information for surgical planning. CT scanning can also help to quantify the size of a Hill-Sachs lesion.

Magnetic resonance imaging (MRI) can provide a great deal of information in cases of instability. Typically an MRI arthrogram is performed after injection of the shoulder using either ultrasound or x-ray guidance. Fig. (2) shows a Bankart lesion found using MRI arthrography and Fig. (3) shows the appearances of a Hill-Sachs lesion on a plain MRI scan. MRI arthrography has been demonstrated to offer increased sensitivity in the detection of anterior labral tears than MRI without contrast [17]. Conventional MRI arthrography would be performed with the patient supine and the arm held by the side in neutral rotation. Alternatively, the shoulder can be placed in the abducted and externally rotated position (ABER) with the palm of the hand resting under the head. There has been a report showing a significant improvement in both the sensitivity and specificity for the detection of anterior labral injuries by utilising the ABER position, with the sensitivity improving from $48 \%$ to $89 \%$ and the specificity from $95 \%$ to $97 \%$ [18]. A more recent and larger study has cast some doubt as to the benefit of the ABER position, showing no differences in the sensitivity or specificity, and so its use remains at the clinician's preference [19].

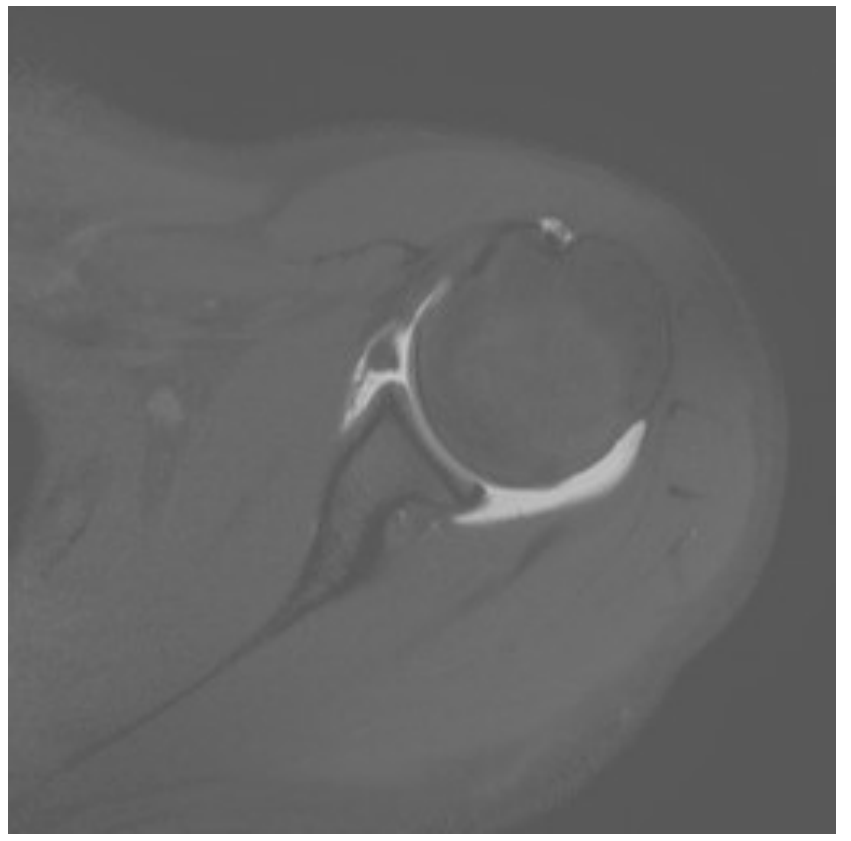

Fig. (2). A Bankart lesion can be seen on this slice from an MR arthrography of the shoulder. Contrast can be seen between the detached anterior labrum and the glenoid.

\section{CLASSIFICATION}

It has proven difficult to produce a comprehensive classification system for shoulder instability capable of defining the relevant aetiology of the condition in each individual. Defining the appropriate treatment for each group has proved even more difficult and has led to an oversimplification in certain systems. There are many elements which could be included in a classification system for shoulder instability, including the direction of instability, whether trauma has been involved, if there is any generalised joint laxity, if there is an underlying neuromuscular or collagen disorder, what structures are involved, if there is abnormal muscle patterning, or if the instability is volitional.

Rockwood's classification system brings together the elements of trauma and volition and combines them into 4 groups [20]. In type 1 instability, there has been a history of traumatic subluxation, but without a frank dislocation. Type 
2 , is as per type 1 , but with a history of a frank dislocation as a primary event. Patients in type 3 describe voluntary subluxation of their shoulders with no history of a traumatic event. This group is subdivided into types $3 \mathrm{a}$ and $3 \mathrm{~b}$, with type $3 \mathrm{a}$ including a history of psychiatric problems and type 3 b not. Finally, type 4 classifies patients with atraumatic involuntary subluxation. The Rockwood classification system has its merits, in the sense that it would be very helpful to be able to place patients into clear categories. Difficulties arise though when the clinical history is not clear, or when trying to determine whether or not there has been a true traumatic dislocation. Distinguishing between a type 3 volitional subluxation and a type 4 involuntary subluxation can also be very difficult and there may be a spectrum where both elements exist. A patient with volitional subluxation could have developed abnormal patterns of muscle recruitment over time and brought themselves gradually from type 3, to type 4 when their volitional subluxation has become involuntary.

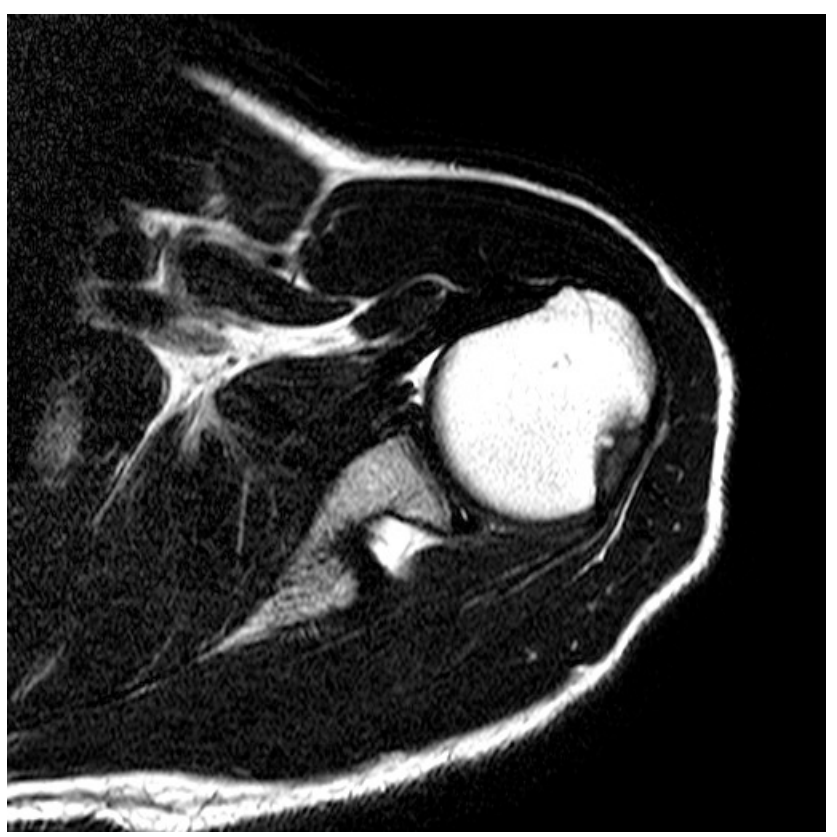

Fig. (3). This MRI scan of the shoulder demonstrates a posterior bony defect of the humeral head (Hill-Sachs lesion).

Kessel and Bayley, have grouped voluntary and involuntary subluxation together, and included both groups under the heading of 'habitual instability' [21]. Kessel and Bayley's terminology is useful when imagining the spectrum, in which voluntary subluxation becomes involuntary and it is this concept of a spectrum of abnormality that provides the basis for the more recent Stanmore classification of shoulder instability [22]. However, when assessing patients, it can be useful to consider the Rockwood type 3 group with purely volitional subluxation, as it is these patients who could be prevented from entering the involuntary group if they are educated in a timely manner to avoid voluntary subluxation, or 'trick movements' and therefore try to avoid the development of ingrained abnormal muscle recruitment patterns [22].

If a classification system is simple, it is often remembered easily and disseminated widely. The classification system described by Thomas and Matsen is one of these systems whose virtue is found in its simplicity [23]. Their paper was a case series describing their technique of open anterior repair and the results. $97 \%$ of their patients had a classic Bankart lesion and $48 \%$ additionally had a large Hill-Sachs lesion. As such they suggested that the majority of their patients would fall into a group they termed TUBS (traumatic, unidirectional, Bankart lesion and surgery). Based on the experience of their unit, but not on published data, they described a second group termed AMBRI (atraumatic, multidirectional, bilateral, rehabilitation and inferior capsular shift). They suggested that patients in the second group are prone to multidirectional instability and should be managed with rotator cuff strengthening. If surgery was to be contemplated in this group, then they suggested that an inferior capsular shift would be the procedure of choice. There has been criticism of their description of the AMBRI group on the basis that it is an over simplification of a complex issue, ignoring the concepts of muscle patterning and of voluntary instability. Furthermore, there has been a suggestion that adopting this classification system may lead to the potential of wrongly operating on patients in the AMBRI group [23]. Thomas and Matsen's paper offered these two terms as a concept, but it is clear that it was not their intention for this to be taken as a comprehensive management algorithm, but perhaps more of a descriptive terminology used in their institution.

Schneeberger and Gerber's classification system encompasses three major facets; the presence of generalised joint laxity, where there was a single major trauma or multiple minor traumas, and whether the instability was unidirectional or multidirectional [24]. This system suggests that the aetiology of all instability is traumatic in nature. Patients with no demonstrable general laxity can either suffer a single traumatic event, developing unilateral instability (similar to Thomas and Matsen's TUBS), or they can be affected by multiple traumatic events and develop multidirectional instability. In the presence of generalised joint laxity, it was suggested that patients could go onto either unidirectional instability or multidirectional instability and that both of these would be as a result of multiple minor repetitive traumatic events which may not be clinically significant in a patient without generalised laxity. Similar to all the classification systems above, with the exception of the Stanmore classification system, is the concept of muscle patterning abnormality. This is the concept that a patient with a muscle patterning abnormality as the precipitating problem, may then go on to develop a structural abnormality after a period of chronic instability. Conversely, a patient with instability secondary to trauma may have an overlay of muscle patterning which has developed secondarily.

Perhaps the most all-encompassing classification system is the Stanmore system [22]. All the classification systems described above attempted to guide management by using strict subdivisions, but the difficulty arose in deciding into which of these subdivisions individual patients should be placed. Without the ability to place all patients into management guiding boxes, the usefulness of a classification system is diminished. The Stanmore system attempts to find a place for all patients, by positioning them at a single point within an elegant looking triangle (see Fig. 4). The concept is that patients can be at one of three poles, in which case 
they will exhibit a defined set of features placing them there. Polar type 1 patients will have a defined history of a significant trauma, display unidirectional instability and have a Bankart lesion. These patients are thought to be equivalent to the TUBS subgroup of the Thomas and Matsen system [23]. Patients at the $2^{\text {nd }}$ pole are similar to those of the AMBRI group described above and have a less defined history of trauma, but are likely to have a structural lesion and do have an overlay of abnormal muscle recruitment (muscle patterning). At the $3^{\text {rd }}$ pole, patients have no structural abnormality and may be habitual dislocators or have a significant muscle patterning abnormality. This system allows patients to move around the triangle over time. For example, a patient in the polar type 2 group may subsequently develop a muscle patterning disorder and shift towards the $3^{\text {rd }}$ pole. The difficulty with this classification system is deciding where to place patients in the triangle. When patients fit into the TUBS category, it is easy to place them at the $1^{\text {st }}$ pole, however, anything other than this becomes complex and in essence explains why Thomas and Matsen described their simple two group model.

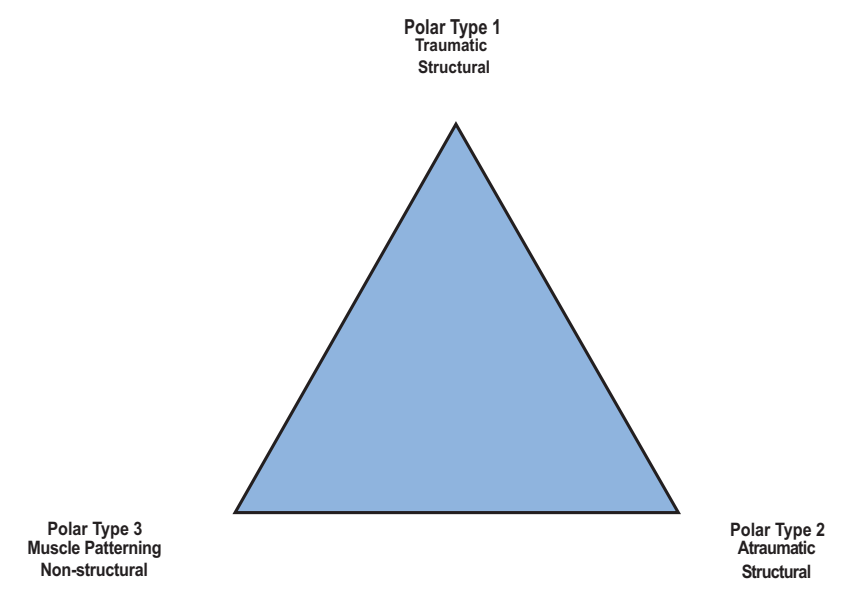

Fig. (4). The Stanmore classification system of shoulder instability.

\section{MANAGEMENT}

When assessing patients who fall into the TUBS group (Stanmore polar type 1), a history of a significant traumatic event is usually elicited and will often be combined with evidence of an anterior dislocation, both from the emergency department records and from the plain radiographs on the day of injury. It is likely that these patients will display a positive anterior apprehension test when examined. An MR arthrogram of the shoulder should confirm that a Bankart lesion is present and there may also be a Hill-Sachs lesion. With this information, it is possible to have a reasonable discussion with patients to produce a management plan. The surgical procedure of choice in our unit for these patients is an arthroscopic Bankart repair. When counselling patients, the variables taken into account are: age, time since injury, success of non-operative treatment so far, the number of recorded dislocations of the shoulder, sporting level, type of work and patient preference.

If a patient in the TUBS group has had a recent injury, plays sport and wishes to consider arthroscopic Bankart repair, then they will want to have an idea of what their redislocation rate is likely to be if they opt for surgery or not.
There are multiple case series demonstrating high rates of redislocation after non-surgical management throughout the last half century. In the 1950s, one such large series of 313 patients (by the time of final follow up) showed a redislocation rate of $38 \%$ at a mean follow up time of 4.8 years [25]. Those patients who were under the age of 20 at the time of initial dislocation had an $83 \%$ chance of experiencing a further dislocation, whereas those who were over the age of 40 had only a $16 \%$ chance, emphasising the importance of patient age when counselling them for surgical management. This was a retrospectively analysed series, but a prospective case series in the 1980s has demonstrated a similar re-dislocation rate of $44 \%$ at 5 years follow up, as well as showing no difference between patients who had been immobilised in a sling for 1 week versus those who had had 3 weeks immobilisation [26]. Interestingly, a Japanese group presented a study which included a group of 20 patients who were immobilised in an external rotation brace (set at 10 degrees external rotation), demonstrating no redislocation [27]. This was based on evidence from MRI scans of the shoulder demonstrating that the detached anterior labrum rested in a more anatomical position when the shoulder joint was externally rotated [28]. The same group followed this up with a randomised controlled trial of 198 patients, comparing internal rotation versus external rotation bracing [29]. At 2 years follow up, the recurrence rate was $42 \%$ in the internal rotation group and $26 \%$ in the external rotation group. This difference did reach significance, but a $26 \%$ recurrence rate may still not be acceptable to many patients and was clearly not as good as the early promise from their pilot study [27]. A further randomised controlled trial of bracing in internal versus external rotation has been published from an independent centre [30]. This was a smaller study, with only 51 patients included, but at 33 months follow up there was no significant difference between the 2 groups in terms of dislocation rate, with a rate of $41.7 \%$ in the internal rotation group and $37 \%$ in the external rotation group. These high rates of redislocation and the lack of effect from the external rotation bracing protocol cast doubt on this method of treatment.

There has been a gradual accumulation of evidence in support of arthroscopic repair of Bankart lesion for patients in the TUBS group. Initially there were promising early reports from retrospective case series of arthroscopic Bankart repair following first time dislocations in young patients with recurrent instability rates ranging from $5 \%$ to $17 \%$ [31-35]. Following on from that, there has been several cohort studies comparing recurrence rates between arthroscopic stabilisation and non operative management [36-39]. All of these studies demonstrated significant reductions in the rate of recurrence with stabilisation. A question has arisen as to whether early arthroscopic lavage alone, without Bankart lesion repair may reduce the risk of further dislocation or symptomatic subluxation. Wintzell compared sling alone against early arthroscopic lavage and demonstrated a reduced recurrence rate of $20 \%$ in the lavage group versus $60 \%$ in the sling group [40]. There were, however only 15 patients in each group, making it difficult to make firm recommendations. Clarification arrived courtesy of a carefully conducted randomised controlled trial from Edinburgy [41]. 44 patients in the control group received arthroscopic lavage and 44 received arthroscopic Bankart 
repair. After 2 years follow up, the rate of re-dislocation or symptomatic recurrence was $7 \%$ in the arthroscopic Bankart repair group and $38 \%$ in the arthroscopic lavage alone group. The patients in the Bankart repair group were also more than 3 times as likely to return to playing sport.

This accumulation of evidence to support arthroscopic Bankart repair has made it possible to effectively counsel patients. Each decision has to be an individualised decision, rather than being an algorithm based treatment model. Some patients will opt for early arthroscopic repair, particularly if they are young and wish to engage in competitive sport. It is equally reasonable to trial a period of non-operative treatment and if there is no on-going symptomatic instability, to defer any further treatment in the knowledge that a further dislocation or recurrent instability may occur at a later date.

If arthroscopic Bankart repair is planned then the technical limitations of the procedure need to be considered. The first of these is if there is a large bony glenoid defect. This is less likely in the first time dislocator, but if there have been several dislocations or a long history of symptomatic instability then a pre-operative CT assessment of the glenoid bone stock may be useful. Further assessment can be made at the time of arthroscopy, with a laterally based portal being particularly useful for this. If a defect of more than $25 \%$ of the diameter of the glenoid is noted, then serious consideration should be paid to utilising a bony procedure, rather than a standard capsulolabral Bankart repair. Burkhart and De Beer analysed their arthroscopic Bankart repairs and found that in the presence of a significant bony glenoid defect (greater than 25\%), that there was a significantly increased risk of further dislocation [42]. At a mean follow up of 27 months, the risk of further dislocation in the group without significant bone loss was $4 \%$, versus $67 \%$ in the group with significant bone loss. Based on these results, the authors moved to using a 'Latarjet' procedure for managing patients who had more than $25 \%$ glenoid bone loss [43]. This procedure involves transferring the tip of the coracoid process, along with the attached conjoined tendon, and fixing it into the bony glenoid defect with screws (see Fig. 5 for post-operative radiographs). It was first described by Latarjet in 1954 [44]. Burkhart and De Beer demonstrated a recurrence rate of $4.2 \%$ in 102 patients, at a mean follow up of 59 months. The excellent results observed in this series emphasise the importance of determining if there is a significant bony defect.
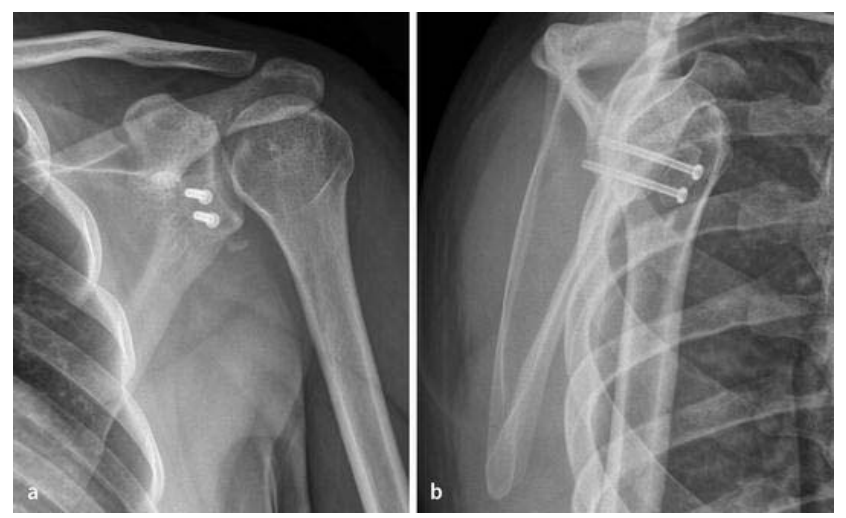

Fig. (5). Radiographs demonstrating the position of the transferred coracoid process after a Latarjet procedure.

Secondly, if there is a large Hill-Sachs lesion, then this should be carefully evaluated. At the time of arthroscopy, the shoulder can be taken through a physiological range of motion and the Hill-Sachs lesion can be observed to determine if it has a tendency to engage with the edge of the glenoid. This is a potential cause of failure of an arthroscopic Bankart repair and at our unit the preferred management is to add a 'remplissage' procedure to the standard arthroscopic Bankart repair. This technique essentially involves suturing the posterior capsule into bony defect [45]. Fig. (6) shows large Hill-Sachs lesion at the time of arthroscopy and Fig. (7) demonstrates the 'remplissage' procedure after the suture anchors have been screwed into the defect and the sutures passed through the posterior capsule.

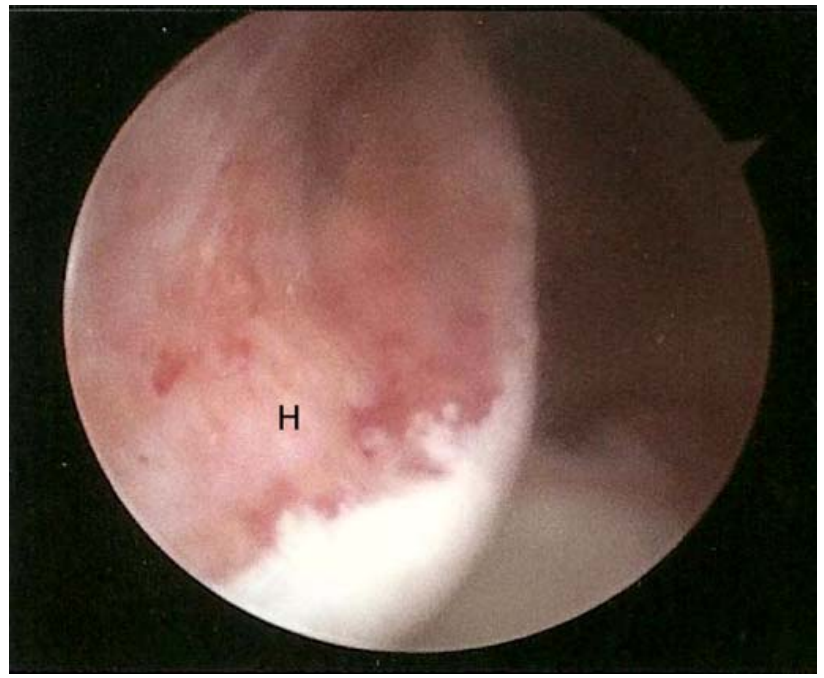

Fig. (6). A large posterior Hill-Sachs defect of the humeral head.

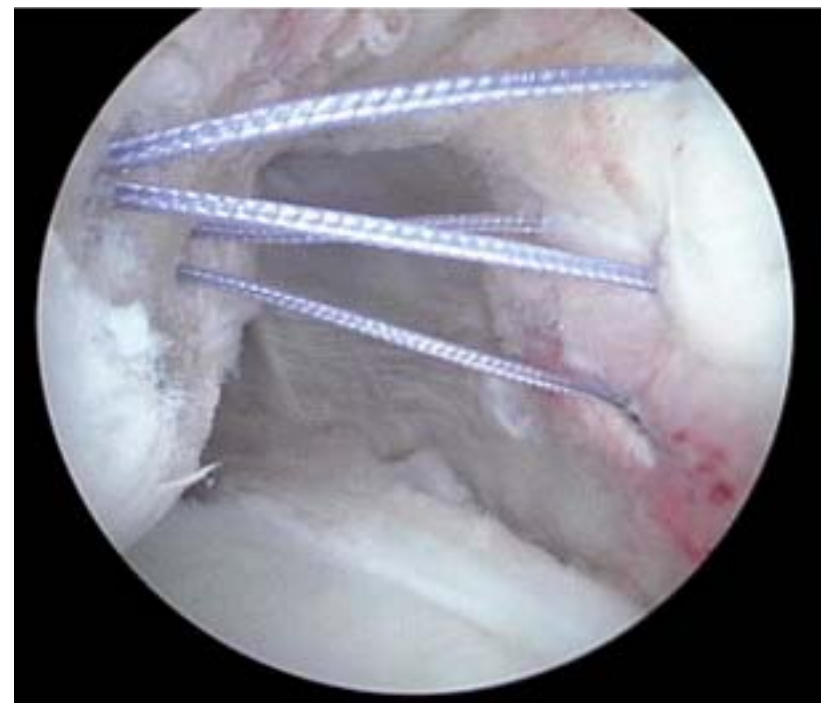

Fig. (7). Sutures have been passed through the posterior capsule from anchors placed into the humeral head as part of a 'Remplissage' procedure. Once these sutures are tied, the posterior capsule will fill the defect.

Thirdly, in cases of traumatic structural anterior instability, there can be a humeral avulsion of the inferior gleno-humeral ligament (HAGL lesion). Fig. (8) demonstrates the appearance of this lesion on MR arthrography of the shoulder. This can be associated with a Bankart lesion, in which case open repair of both lesions are 
indicated and has been described [46]. Typically a HAGL lesion would be repaired using an open technique, but a technically demanding arthroscopic technique has been described [47].

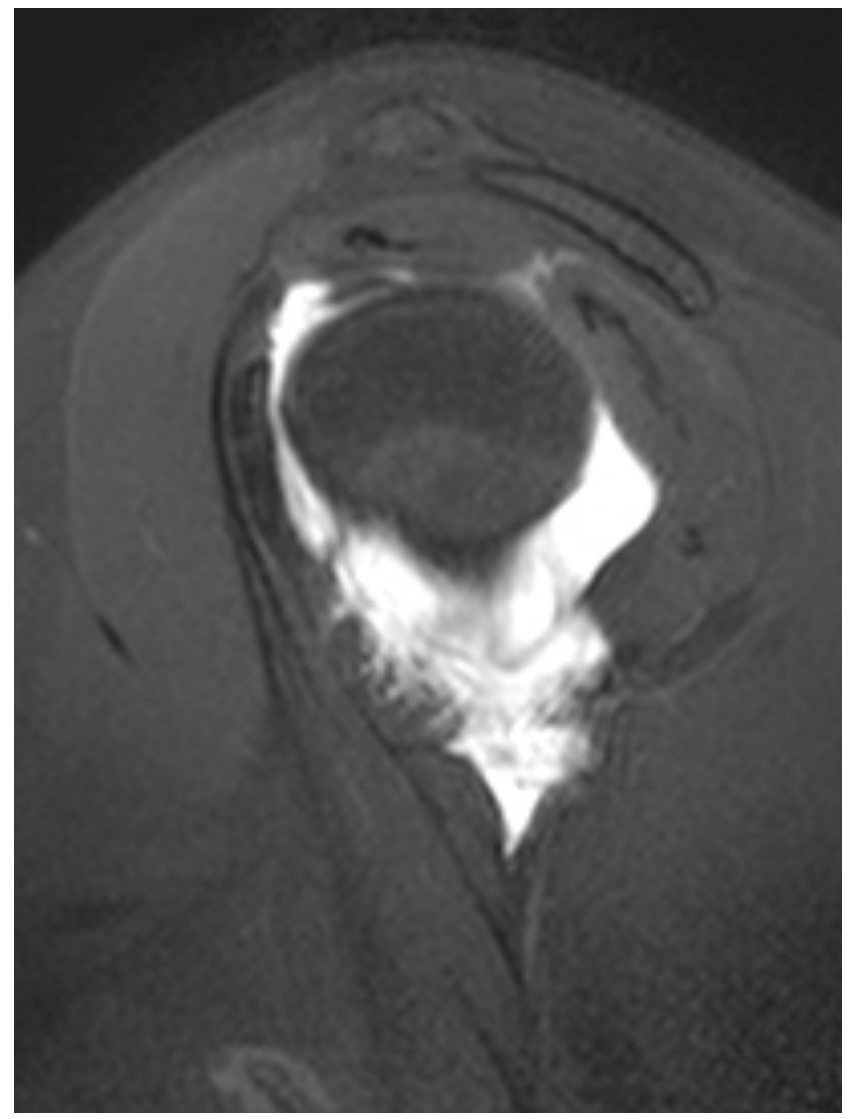

Fig. (8). A humeral avulsion of the gleno-humeral ligament (HAGL) lesion seen on a sagittal slice from an MR arthrogram of the shoulder.

For patients in the AMBRI group, whether they fall into the Stanmore polar group 2 or group 3, a determined attempt at non-operative management is always indicated first. This should be delivered by physiotherapists with specialist experience in this area. Surgery should only be considered if there is a defined structural defect detected on imaging or arthroscopy and only after an initial period of physiotherapy. If there is no structural defect and the main pathology is suspected to be abnormal muscle recruitment (muscle patterning, Stanmore group 3) then surgery is contraindicated. It can be very difficult to ascertain the degree of muscle patterning abnormality in individual patients and hence difficult to determine where to place them on the Stanmore triangle. There is currently interest in the use of electro-myographic analysis (EMG) to help understand the complexities of muscle patterning, but its use is currently experimental [48]. Patients, who fall into the Stanmore group 2, may have unidirectional instability requiring Bankart repair, if they have failed non-operative therapy, or they may have multidirectional instability with capsular dysfunction. In the later situation, an inferior capsular shift would be the procedure of choice. A Bankart repair can also be combined with a capsular shift, if the appropriate lesion is present. The capsular shift reduces capsular volume when there is redundant inferior capsule and can be performed arthroscopically with reasonable results. Treacy and colleagues found that the rate of recurrent instability after arthroscopic capsular shift for multidirectional instability was $12 \%$ at 2 years [49]. Good results have also been observed using an open inferior capsular shift with a recurrence rate of only $4 \%$ after an mean follow up of 61 months [50]. An argument can be made to use either an arthroscopic or open technique to perform an inferior capsular shift. In the absence of a high quality trial to compare the two procedures, it is not possible to firmly recommend one over the other.

\section{CONCLUSION}

When managing patients with shoulder instability, a careful and methodical clinical assessment is a pre-requisite to guide the appropriate management. The range of classification systems can be difficult to utilise in all cases, but knowledge of their principles can help to determine which patients are likely to benefit from surgery. More work is needed to understand how to assess and rehabilitate patients who have muscle patterning abnormalities.

\section{CONFLICT OF INTEREST}

The authors confirm that this article content has no conflict of interest.

\section{ACKNOWLEDGEMENTS}

Declared none.

\section{REFERENCES}

[1] Warner JJ, Deng XH, Warren RF, Torzilli PA. Static capsuloligamentous restraints to superior-inferior translation of the gleno-humeral joint. Am J Sports Med 1992; 20: 675-85.

[2] Budoff JE. In: Trumble TE, Budoff JE, Cornwall R Eds. Core Knowledge in Orthopaedics, Hand, Elbow and Shoulder. PA: Elsevier Science 2006.

[3] Warner JJP, Deng X, Warren RF, Torzilli PA, O'Brien SJ Superoinferior translation in the intact and vented glenohumeral joint. J Shoulder Elbow Surg 1993; 2: 99-105.

[4] Itoi E, Hsu HS, An KN: Biomechanical investigation of the glenohumeral joint. J Shoulder Elbow Surg 1996; 5: 407-24.

[5] O'Brien SJ, Neves MC, Arnoczky SP, et al. The anatomy and histology of the inferior gleno-humeral ligament complex of the shoulder. Am J Sports Med 1990; 18: 449-56.

[6] Rowe CR, Zarins B. Recurrent transient anterior subluxation of the shoulder. J Bone Joint Surg Am 1981; 63A: 863-72.

[7] Beighton PH, Horan F. Orthopaedic aspects of the Ehlers-Danlos syndrome. J Bone Joint Surg Br 1969; 51: 444-53.

[8] Jobe FW, Moynes DR. Delineation of diagnostic criteria and a rehabilitation program for rotator cuff injuries. Am J Sports Med 1982; 10: 336-9.

[9] Gerber C, Krushell RJ. Isolated rupture of the tendon of the subscapularis muscle. Clinical features in 16 cases. J Bone Joint Surg Br 1991; 73B: 389-94.

[10] Neer CS, Foster CR. Inferior capsular shift for involuntary inferior and multidirectional instability of the shoulder. J Shoulder Elbow Surg 2001; 10: 68-72.

[11] Lo IK, Nonweiler B, Woolfrey M, Litchfield R, Kirkley A. An evaluation of the apprehension, relocation and surprise tests for anterior shoulder instability. Am J Sports Med 2004; 32: 301-7.

[12] Speer KP, Hannafin JA, Altchek DW, Warren RF. An evaluation of the shoulder relocation test. Am J Sports Med 1994; 22: 177-83.

[13] O'Driscoll SW. A reliable and simple test for posterior instability of the shoulder. J Bone Joint Surg Br 1991; 73B (Suppl 1): 50.

[14] Bohnsack M, Wulker N. Mini-Symposium: Shoulder Problems (IV) Shoulder Instability. Curr Orthop 2002; 16: 32-40.

[15] Bankart ASB. The pathology and treatment of the recurrent dislocations of the shoulder joint. Br J Surg 1938; 26: 23-9. 
[16] Hill HA, Sachs MD. The grooved defect of the humeral head: A frequently unrecognised complication of dislocations of the shoulder joint. Radiology 1940; 35: 690-700.

[17] Magee T. 3-T MRI of the shoulder: is MR arthrography necessary? Am J Roentgenol 2009; 192: 86-92.

[18] Cvitanic O, Tirman PF, Feller JF, et al. Using abduction and external rotation of the shoulder to increase the sensitivity of MR arthrography in revealing tears of the anterior glenoid labrum. Am J Roentgenol 1997; 169: 837-44.

[19] Schreinemachers SA, Van der Hulst VP, Jaap Willems W, Bipat S, Van der Woude HJ. Is a single direct MR arthrography series in ABER position as accurate in detecting anteroinferior labroligamentous lesions as conventional MR arthrography? Skeletal Radiol 2009; 38: 674-83.

[20] Rockwood CA. Subluxation of the shoulder: the classification, diagnosis and treatment. Orthop Trans 1979; 4: 306.

[21] Kessel L, Bayley JIL Clinical disorders of the shoulder. $2^{\text {nd }}$ ed. USA: Churchill Livingstone, 1986; pp. 189-97.

[22] Lewis A, Kitamura T, Bayley JIL. The classification of shoulder instability: new light through old windows! Curr Orthop 2004; 18: 97-108.

[23] Thomas SC, Matsen FA. An approach to the repair of avulsion of the glenohumeral ligaments in the management of traumatic anterior glenohumeral instability. J Bone Joint Surg Am 1989; 71: 506-13.

[24] Schneeberger AG, Gerber C. Classification and therapy of the unstable shoulder. Ther Umsch 1998; 553: 187-91.

[25] Rowe CD. Prognosis in dislocations of the shoulder. J Bone Joint Surg Am 1956; 38A: 957-77.

[26] Hovelius L. Anterior dislocation of the shoulder in teenagers and young adults: Five year prognosis. J Bone Joint Surg Br 1987; 69A: 393-9.

[27] Itoi E, Hatakeyama Y, Kido T, Sato T, Minagawa H, Wakabayashi I, Kobayashi M. A new method of immobilisation after traumatic anterior dislocation of the shoulder: a preliminary study. J Shoulder Elbow Surg 2003; 12: 413-5.

[28] Itoi E, Sashi R, Minagawa H, Shimizu T, Wakabayashi I, Sato K. Position of immobilisation after dislocation of the glenohumeral joint: a study with the use of magnetic resonance imaging. J Bone Joint Surg Am 2001; 83A: 661-7.

[29] Itoi E, Hatakeyama Y, Sato T, et al. Immobilization in external rotation after shoulder dislocation reduces the risk of recurrence: A randomized controlled trial. J Bone Joint Surg Am 2007; 89: 12431.

[30] Finestone A, Milgrom C, Radeva-Petrova DR, et al. Bracing in external rotation for traumatic anterior dislocation of the shoulder. $\mathbf{J}$ Bone Joint Surg Br 2009; 91B: 918-21.

[31] Uribe JW, Hechtman KA. Arthroscopically assisted repair of acute Bankart lesion. Orthopaedics 1993; 16: 1019-23.

[32] Arciero RA, Taylor DC, Snyder RJ, Uhorchak JM. Arthroscopic bioabsorbable tack stabilisation of initial anterior shoulder dislocations: A preliminary report. Arthroscopy 1995; 11: 410-17.

[33] Salmon JM, Bell SN. Arthroscopic stabilisation of the shoulder for acute primary dislocations using a transglenoid suture technique. Arthroscopy 1998; 14: 143-7.

[34] Valentin A, Winge S, Engstrom B. Early arthroscopic treatment of primary anterior shoulder dislocation. A follow up study. Scand $\mathbf{J}$ Med Sci Sports 1998; 8: 405-10.
[35] Boszotta H, Helperstorfer W. Arthroscopic transglenoid suture repair for initial anterior shoulder dislocation. Arthroscopy 2000; 16: 462-70.

[36] Wheeler JH, Ryan JB, Arciero RA, Molinari RN. Arthroscopic versus non-operative treatment of acute shoulder dislocations in young athletes. Arthroscopy 1989; 5: 213-7.

[37] Arciero RA, Wheeler JH, Ryan JB, McBride JT. Arthroscopic Bankart repair versus non-operative treatment for acute, initial anterior shoulder dislocations. Am J Sports Med 1994; 22: 589-94.

[38] DeBarardino TM, Arciero RA, Taylor DC, Uhorchak JM. Prospective evaluation of arthroscopic stabilization of acute, initial anterior shoulder dislocations in young athletes. Two to five year follow up. Am J Sports Med 2001; 29: 586-92.

[39] Larrain MV, Botto GJ, Montenegro HJ, Mauas DM. Arthroscopic repair of acute traumatic anterior shoulder dislocation in young adults. Arthroscopy 2001; 23: 119-23.

[40] Wintzell G, Haglund-Akerlind Y, Ekelund A, Sandstrom B, Hovelius L, Larsson S. Arthrosopic lavage compared with nonoperative treatment for traumatic primary anterior shoulder dislocation: a 2 year follow up of a prospective randomized study. J Shoulder Elbow Surg 1999; 8: 399-402.

[41] Robinson CM, Jenkins PJ, White TO, Ker A, Will E. Primary arthroscopic stabilisation for a first time anterior dislocation of the shoulder. A randomised, double-blind trial. . J Bone Joint Surg Am 2008; 90A: 708-21.

[42] Burkhart SS, De Beer JF. Traumatic gleno-humeral bone defects and their relationship to failure of arthroscopic Bankart repairs: significance of the inverted-pear glenoid and the humeral head engaging Hill-Sachs lesion. Arthroscopy 2000; 16: 677-94.

[43] Burkhart SS, De Beer JF, Barth JR, Cresswell T, Roberts C, Richards DP. Results of the modified Latarjet reconstruction in patients with anterio-inferior instability and significant bone loss Arthroscopy 2007; 23: 1033-41.

[44] Latarjet M. Treatment of recurrent dislocation of the shoulder. Lyon Chir 1954; 49: 994-7.

[45] Purchase RJ, Wolf EM, Hobgood ER Hill-Sachs 'Remplissage': An arthroscopic solution for the engaging Hill-Sachs lesion. Arthroscopy 2008; 24: 723-6.

[46] Bhatia DN. Dual-window subscapularis-sparing approach: a new surgical technique for combined reconstruction of a glenoid bone defect or Bankart lesion associated with a HAGL lesion in anterior shoulder instability. Tech Hand Up Extrem Surg 2012; 16: 30-6.

[47] Richards DP, Burkhart SS. Arthroscopic humeral avulsion of the glenohumeral ligaments (HAGL) repair. Arthroscopy 2004; 20 (Suppl 2): 134-41.

[48] Jaggi A, Malone AA, Cowan J, Lambert S, Bayley I, Cairns MC. Prospective blinded comparison of surface versus wire electromyographic analysis of muscle recruitment in shoulder instability. Physiother Res Int 2009; 14: 17-29.

[49] Treacy SH, Savoie FH, Field LD. Arthroscopic treatment of multidirectional instability. J Shoulder Elbow Surg 1999; 8: 34550 .

[50] Pollock RG, Owens JM, Flatow EL, Bigliani LU. Operative results of the inferior capsular shift procedure for multi-directional instability of the shoulder J Bone Joint Surg Am 2000; 82A: 91928. 